\title{
PRESCRIBED HOLONOMY FOR PROJECTIVE STRUCTURES ON COMPACT SURFACES
}

\author{
DANIEL M. GALLO
}

1. Introduction. Let $S$ be a compact oriented surface of genus $g \geq 2$. Let $U$ be the universal cover of $S$ and denote the Riemann sphere by $\hat{\mathbf{C}}$. The group of $2 \times 2$ complex matrices with determinant equal to 1 will be denoted by $S L(2, \mathrm{C})$. The quotient group $\operatorname{PSL}(2, \mathrm{C})=S L(2, \mathrm{C}) /\{ \pm \mathrm{id}\}$ is canonically isomorphic to the group of conformal automorphisms of $\hat{\mathbf{C}}$.

A projective structure on $S$ consists of

(i) an orientation preserving local homeomorphism (or developing map) $f: U \rightarrow \hat{\mathbf{C}}$, and

(ii) a homomorphism (or holonomy map) $\phi: \pi_{1}(S) \rightarrow \operatorname{PSL}(2, \mathrm{C})$ such that $f \circ \gamma(x)=\phi(\gamma) \circ f(x)$ for all $\gamma \in \pi_{1}(S), x \in U$.

Identifying $\hat{\mathbf{C}}$ with the boundary of the open unit ball $B^{3} \subset \mathbf{R}^{3}$, there is a natural extension of the action of $\operatorname{PSL}(2, \mathbf{C})$ on $\hat{\mathbf{C}}$ to an action on $B^{3} \cup \hat{\mathbf{C}}$. A subgroup $G$ of $P S L(2, \mathrm{C})$ will be called elementary if there is a subset of $B^{3} \cup \hat{\mathbf{C}}$ consisting of one or two points which is invariant under $G$.

The purpose of this note is to announce

THEOREM 1. Let $\phi: \pi_{1}(S) \rightarrow \operatorname{PSL}(2, \mathrm{C})$ be a homomorphism which lifts to $S L(2, \mathrm{C})$ with $\phi\left(\pi_{1}(S)\right)$ nonelementary. Then $\phi$ is the holonomy of a projective structure on $S$.

It is well known that the converse is true. More precisely, if $\phi$ is the holonomy of a projective structure on $S$ then

(i) $\phi\left(\pi_{1}(S)\right)$ is nonelementary (see [6]), and

(ii) $\phi$ lifts to $S L(2, \mathrm{C})$ (see $[4,5])$.

Classically, the theory of projective structures on compact surfaces has been closely related to problems concerning uniformization and discontinuous groups. The names of Klein and Poincaré stand out in this context. In modern times the work of Ahlfors and Bers has revitalized interest in the theory and more recently the geometric, topological methods of Thurston $[8,9]$ have shed new light on it. Finally, we note that Theorem 1 was conjectured by Thurston. For more details on the history of the subject see Gunning [5] or Hejhal [6] and the references therein.

In the special case that $\phi\left(\pi_{1}(S)\right) \subset \operatorname{PSL}(2, \mathbf{R})$, Theorem 1 was proved by Gallo, Goldman and Porter [1]. If $\phi\left(\pi_{1}(S)\right)$ has an invariant circle (for its action on $\hat{\mathbf{C}}$ ) then it is conjugate to a finite extension of a subgroup of $\operatorname{PSL}(2, \mathbf{R})$. Consequently, in this case, a slight modification of the methods found in [1] can be used to prove the theorem.

Received by the editors January 22, 1988 and, in revised form, May 24, 1988.

1980 Mathematics Subject Classification. Primary 30F10; Secondary 57M99. 
We give a brief outline of the proof for the remaining case.

2. The $S L(2, \mathrm{C})$ condition. Given a homomorphism

$$
\phi: \pi_{1}(S) \rightarrow P S L(2, \mathrm{C})
$$

there is a natural action of $\pi_{1}(S)$ on $U \times \hat{\mathbf{C}}$ (for $\gamma \in \pi_{1}(S)$ and $(x, y) \in U \times \hat{\mathbf{C}}$, $\gamma(x, y)=(\gamma(x), \phi(\gamma)(y)))$ and the suspension $E_{\phi}=(U \times \hat{\mathbf{C}}) / \pi_{1}(S)$ is a sphere bundle over $S$. These bundles are characterized topologically by their Stiefel-Whitney classes $W_{\phi} \in H^{2}\left(S, \mathbf{Z}_{2}\right)$.

For $P \subset S$ an open surface we define (except in some degenerate cases) the relative Stiefel-Whitney class $W_{\phi, P} \in H^{2}\left(P, \partial P, \mathbf{Z}_{2}\right)$ by specifying canonical sections from $\partial P$ into the principal bundle associated to $E_{\phi}$. This is the natural generalization of the relative Euler class defined by Goldman $[\mathbf{1 , 2 , 3 ]}$.

An element of $\operatorname{PSL}(2, \mathrm{C})$ is called loxodromic with rotation angle $\theta$ if it is conjugate to a transformation of the form $\gamma(z)=r e^{i \theta} z$ with $r>1$ and $-\pi<\theta \leq \pi$ (if $\theta=0$ the element will be called hyperbolic).

Now let $P \subset S$ be a pair of pants and identify $\pi_{1}(P)$ with a subgroup of $\pi_{1}(S)$. Thus $\pi_{1}(P)=\left\langle\gamma_{1}, \gamma_{2}\right\rangle$ with $\gamma_{1}, \gamma_{2} \in \pi_{1}(S)$. A path of homomorphisms $\phi_{t}:\left\langle\gamma_{1}, \gamma_{2}\right\rangle \rightarrow P S L(2, \mathbf{C})$ with $t \in[0,1]$ will be called a deformation if for $k=1,2, \phi_{t}\left(\gamma_{k}\right)$ and $\phi_{t}\left(\gamma_{1} \circ \gamma_{2}\right)$ (hence $\phi_{t}\left(\gamma_{2} \circ \gamma_{1}\right)$ also) are loxodromic with rotation angle different from $\pi$ for all $t$.

A homomorphism $\phi:\left\langle\gamma_{1}, \gamma_{2}\right\rangle \rightarrow \operatorname{PSL}(2, \mathbf{R})$ is of type 1 if $\phi\left(\gamma_{1}\right), \phi\left(\gamma_{2}\right)$, $\phi\left(\gamma_{1} \circ \gamma_{2}\right)$, and $\phi\left(\gamma_{2} \circ \gamma_{1}\right)$ are hyperbolic with axes oriented as in Figure 1 (or Figure 1 with the arrows reversed).

To prove Theorem 1 we use

Proposition 1. Let $\phi: \pi_{1}(S) \rightarrow P S L(2, \mathrm{C})$ be a nonelementary homomorphism which lifts to $S L(2, \mathbf{C})$. Then there exists a pants decomposition $P_{1}, \ldots, P_{2 g-2}$ of $S$ with the following properties:

(i) $\phi(\gamma)$ is loxodromic for each $\gamma \in \pi_{1}(S)$ homotopic to a boundary component of (some) $P_{j}$;

(ii) (here $\phi_{j}$ will denote the restriction of $\phi$ to $\left.\pi_{1}\left(P_{j}\right)\right), \phi_{j}:\left\langle\gamma_{j, 1}, \gamma_{j, 2}\right\rangle \rightarrow$ $\operatorname{PSL}(2, \mathrm{C})$ is an isomorphism onto a Schottky group with free generators $\phi_{j}\left(\gamma_{j, 1}\right)=\gamma_{j, 1}^{\prime}, \phi_{j}\left(\gamma_{j, 2}\right)=\gamma_{j, 2}^{\prime} ;$

(iii) $W_{\phi, P_{j}}=1$;

(iv) $\phi_{j}: \pi_{1}\left(P_{j}\right) \rightarrow \operatorname{PSL}(2, \mathrm{C})$ is deformable to a type 1 isomorphism.

The proposition is proved by applying Dehn twists to the surface $S$. The proofs of (i) and (ii) use only the fact that $\phi\left(\pi_{1}(S)\right)$ is nonelementary. To prove (iii) and (iv) one needs that

$$
\sum_{j=1}^{2 g-2} W_{\phi, P_{j}}=\bmod 2 W_{\phi}=0 .
$$

The first equality in (1-1) follows from the addition formula in elementary obstruction theory (see Steenrod [7]). The second equality follows from the assumption that $\phi$ lifts to $S L(2, \mathrm{C})$. 


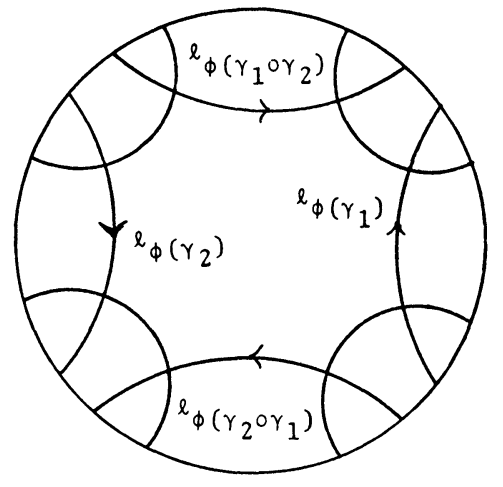

Figure 1

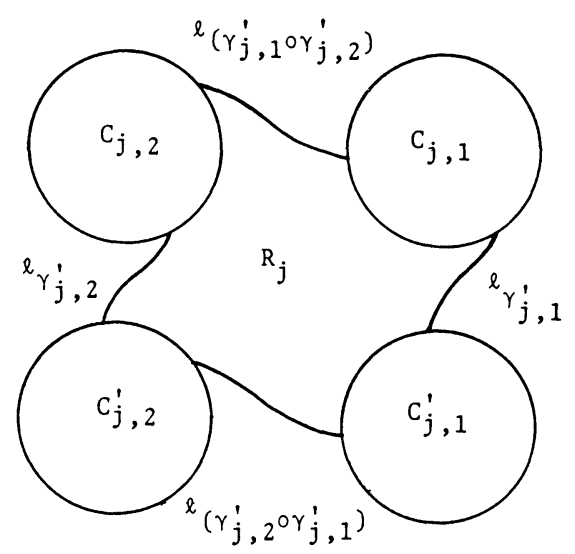

FIGURE 2

(In these figures $l_{\gamma}$ denotes a canonical curve for the Mobius transformation $\gamma$.)

3. The developing map. Let $\gamma(z)=\lambda z$ with $\lambda=r e^{i \theta}, r>1$ and $-\pi<$ $\theta \leq \pi$. The curve $c_{\lambda}: \mathbf{R} \rightarrow \mathbf{C}-\{0\}$ given by $c_{\lambda}(s)=\lambda^{s}$ is invariant under $\gamma$. A simple curve $c: \mathbf{R} \rightarrow \mathbf{C}-\{0\}$ (invariant under $\gamma$ ) will be called canonical (for $\gamma$ ) if $\lim _{s \rightarrow \infty} c(s)=\infty, \lim _{s \rightarrow-\infty} c(s)=0$ and the simple closed curve $c(\mathbf{R}) /\langle\gamma\rangle$ is homotopic (in the torus $(\mathbf{C}-\{0\}) /\langle\gamma\rangle$ ) to $c_{\lambda}(\mathbf{R}) /\langle\gamma\rangle$. It is clear how to define canonical curves for arbitrary loxodromic elements.

Now choose $P_{1}, \ldots, P_{2 g-2}$ a pants decomposition of $S$ as in Proposition 1. Let $\pi: U \rightarrow S$ be the natural projection onto $U / \pi_{1}(S)=S$. Let $U_{j} \subset U$ be a component of $\pi^{-1}\left(P_{j}\right)$ invariant under $\pi_{1}\left(P_{j}\right)=\left\langle\gamma_{j, 1}, \gamma_{j, 2}\right\rangle$ and let $C_{j, k}, C_{j, k}^{\prime}$ be (mutually) disjoint circles with $\gamma_{j, k}^{\prime}\left(C_{j, k}\right)=C_{j, k}^{\prime}$ (Figure 2) which realize $\phi_{j}\left(\pi_{1}\left(P_{j}\right)\right)$ as a Schottky group.

Since $\phi_{j}$ is deformable to a type 1 isomorphism it is easy to show that one may choose a region $R_{j} \subset \hat{\mathbf{C}}$ bounded by arcs on the circles $C_{j, k}, C_{j, k}^{\prime}$ and arcs on canonical curves for the elements $\gamma_{j, 1}^{\prime}, \gamma_{j, 2}^{\prime}, \gamma_{j, 1}^{\prime} \circ \gamma_{j, 2}^{\prime}, \gamma_{j, 2}^{\prime} \circ \gamma_{j, 1}^{\prime}$ such that $\bar{R}_{j} / \phi_{j}\left(\pi_{1}\left(P_{j}\right)\right)$ is a pair of pants (Figure 2).

The existence of the region $R_{j}$ allows one to define a homeomorphism $f_{j}: U_{j} \rightarrow \hat{\mathbf{C}}$ which is a developing map for $\phi_{j}: \pi_{1}\left(P_{j}\right) \rightarrow \operatorname{PSL}(2, \mathbf{C})$. The map $f_{j}$ extends to the boundary components of $U_{j}$ sending them onto canonical curves. On the basis of this last fact one may use cutting and pasting techniques similar to those in [1] to obtain a developing map $f: U \rightarrow \hat{\mathbf{C}}$ for the homomorphism $\phi$. Full details will appear elsewhere.

The author is indebted to W. Goldman for introducing him to Thurston's approach to projective structures and for his constant reminder that Theorem 1 should be true. 


\section{REFERENCES}

1. D. M. Gallo, W. Goldman and R. M. Porter, Projective structures with monodromy in $\operatorname{PSL}(2, \mathbf{R})$ (to appear).

2. W. Goldman, Discontinuous groups and the Euler class, Doctoral Thesis, Univ. of California, Berkeley, 1980.

3. _ Projective structures with Fuchsian holonomy, J. Differential Geom. 25 (1987), 297-326.

4. R. C. Gunning, Lectures on vector bundles over Riemann surfaces, Princeton Univ. Press, Princeton, N.J., 1967.

5 . Affine and projective structures on Riemann surfaces, Riemann Surfaces and Related Topics, Proceedings of the 1978 Stony Brook Conference, (I. Kra and B. Maskit, eds.), Annals of Math. Studies, Princeton Univ. Press, Princeton, N.J. 1981, pp. 225-244.

6. D. Hejhal, Monodromy groups and linearly polymorphic functions, Acta Math. 135 (1976), 215-264.

7. N. Steenrod, The topology of fiber bundles, Princeton Univ. Press, Princeton, N.J., 1951.

8. W. Thurston, The geometry and topology of three-manifolds, Princeton Univ. Lecture Notes.

9. __ Three dimensional manifolds, Kleinian groups and hyperbolic geometry, Bull. Amer. Math. Soc. (N.S.) 6 (1982), 357-381.

Department of Mathematics, Rutgers University, Newark, New Jersey 07102

Current address: Department of Mathematics, St. John's University, Staten Island, New York 10301 